\title{
An Integrated Fuzzy Multi-Criteria Decision-Making Approach for Six Sigma Project
}

\author{
Selection \\ Selçuk Perçin* \\ Department of Business Administration, \\ Karadeniz Technical University, 61080, Trabzon, Turkey \\ Cengiz Kahraman \\ Department of Industrial Engineering, \\ Istanbul Technical University, 34367, Macka, Istanbul, Turkey \\ Received: 14-09-2009 \\ Accepted: 09-09-2010
}

\begin{abstract}
Selecting an appropriate six sigma project is one of the most important problems for a manufacturing company because it impacts the manufacturing costs. Besides, an appropriate choice of Six Sigma projects is very important as it helps to improve quality, reduce variation, and eliminate waste of a manufacturing system. Therefore, the main purpose of this study is to provide a good insight into the use of an integrated decision-making methodology in the evaluation of Six Sigma projects. For this purpose, we integrate three multi-criteria decision-making (MCDM) methods by applying a modified Delphi method, Analytical Hierarchy Process (AHP) and Fuzzy Technique for Order Preference by Similarity to Ideal Solution (FTOPSIS) methodologies. After the evaluation criteria of Six Sigma projects are determined by a Modified Delphi method, the weights of criteria are calculated by applying the AHP method. The FTOPSIS method is then employed to achieve the final ranking results. A real case application along with a sensitivity analysis is presented to show the effectiveness of the proposed methodology. The research question for this methodology is how we can develop an integrated MCDM model to select six sigma projects.
\end{abstract}

Keywords: Six Sigma, Delphi Method, Analytical Hierarchy Process (AHP), Fuzzy TOPSIS, Multi-Criteria Decision-Making (MCDM), Fuzzy Sets

\section{Introduction}

In today's environment, organizations are searching for new business practices and solutions that can provide them with success. Six sigma is one of these methodologies that can lead to greater competitiveness in the market. Six sigma indicates an ultimate goal of less than 3.4 defects per million opportunities (DPMO) for every process (Linderman et al., 2003). Therefore, six sigma is described as a business improvement strategy that seeks to reduce process variations and defects rate in all critical business process to achieve continuous improvements in process performance that impact the bottom line results of an organization (Antony, 2004). Six sigma can also be defined as a highly disciplined and statistically based method that aims at achieving perfection in every process and product of a company (Hahn et al., 2000). Nowadays, six sigma has become one of the most important subjects in business strategy. Thus, six sigma has received considerable attention from researchers. Three research areas for six sigma have attracted interest among the researchers: First, the critical success factors for six sigma implementation, second, the basic concepts, tools, and applications of six sigma approach and finally the selection of six sigma projects for business improvement. This paper focuses on the last area.

Six sigma is regarded as a project-based methodology that can help a company achieve expected benefits through continuous project improvement ( $\mathrm{Su} \&$ Chou, 2008). It is originated at Motorola in the early 1980s, and then many companies such as GE, Boeing, DuPont, Toshiba, Allied Signal, Polaroid, Ford, Honeywell, Texas Instruments, Sony, etc., have

${ }^{*}$ Corresponding author. Tel: +904623773473; fax: +904623257281; E-mail address: selcukpercin@yahoo.com 
reported significant benefits as a result of Six Sigma project implementation (Kwak \& Anbari, 2006; Pande et al., 2000). Today, Six Sigma projects have been evaluated by many organizations as a rapidly expanding source of cost savings, quality improvements, competitive advantage, and customer satisfaction (Hahn et al., 1999; Hendricks \& Kelbaugh, 1998; Motwani et al., 2004). Despite their popularity in all business sectors, however, a significant number of Six Sigma projects fail; many produce only local improvements (Pyzdek, 2000) and about \%50 of them fail to deliver desired results (Zimmerman and Weiss, 2005). Besides the inherent risks, other reasons may be attributed to the complexity, long implementation times, and high operational costs of the implementation projects that may significantly exceed initial capital estimates. In addition, having incompatible objectives aligning with the organization's goals and expectations will also result in failure of six sigma implementation. The implementation of a six sigma project requires commitment of resources, time, money and effort from the entire organization. For this reason, selecting and prioritizing the critical six sigma projects, are real challenges in practice (Coronado \& Antony, 2002; Pande et al., 2000). On the other hand, the current literature fails to demonstrate a real case application showing how six sigma projects are selected in organizations. In other words, there still lacks a wellstructured approach to assist a company in selecting the most suitable projects. For this reason, this study proposes an analytical approach to effectively select Six Sigma projects.

Six sigma project selection can be viewed as a complex multi-criteria decision problem due to the availability of quantitative, qualitative, and multiple criteria that have to be considered in the decisionmaking process. In addition, evaluation procedures involve several objectives and it is often necessary to compromise among these possibly complex and conflicting objectives. For these reasons, multi-criteria decision-making (MCDM) has been found to be a useful approach to solve this kind of problem. Classical MCDM methods assuming that all the weights of evaluation criteria are crisp values can not effectively handle problems with such qualitative and often subjective data. In such situations, the use of fuzzy set approach explicitly handles vague and imprecise data in the decision model. In addition, fuzzy set approaches to decision-making are usually most appropriate when expert evaluations are needed (Kahraman \& Tolga, 2009). In this paper, this is realized by adopting a Modified Delphi Method with the cooperation of multiple experts to identify evaluation criteria. After determining the criteria, the six sigma project selection problem has been formulated by using an integrated methodology including Analytic Hierarchy Process (AHP) and Fuzzy Technique for Order Preference by Similarity to Ideal Solution (FTOPSIS). In other words, AHP is applied to determine the relative weights of evaluation criteria and FTOPSIS is applied to rank the potential six sigma project alternatives. By proposing an integrated fuzzy MCDM model, this paper will not only assist managers in making better decisions in evaluating decision criteria, but also will help them for selecting the best six sigma project.

The paper is organized in five sections. The evaluation framework and existing methods for six sigma project selection is explained in Section 2. The details of the Modified Delphi, AHP and FTOPSIS methods are also given in Section 2. Section 3 presents the application of the developed model to a case company. Section 4 provides discussion and limitations. Finally, the last section contains some concluding remarks and perspectives.

\section{Evaluation Framework and Methods for Six Sigma Project Selection}

Analytical models for six sigma project evaluation and selection range from simple project assessment techniques to complex statistical and mathematical programming approaches. The most common analytical approaches for six sigma project selection include different project assessment techniques such as project assessment matrix (Breyfogle et al., 2001), project selection matrix (Kelly, 2002) and project ranking matrix (Adams et al., 2003). In addition, several statistical techniques for the selection and prioritization of six sigma projects include different statistical tools such as cost-benefit analysis, design of experiments (DOE), statistical process control (SPC), cause-andeffect matrix, brainstorming, Pareto analysis, and Failure Mode and Effects Analysis (FMEA) (Banuelas et al., 2006), and mathematical programming approaches such as Quality Function Deployment 
(QFD) (Pande et al., 2000; Pyzdek, 2003), Analytic Hierarchy Process (AHP) (Banuelas \& Antony, 2003), Data Envelopment Analysis (DEA) (Kumar et al., 2007), and un-weighted scoring models (Banuelas et al., 2006).

As described above, most of these methods have been developed based on the concepts of accurate measurements and crisp evaluation. However, the evaluation process of six sigma project selection involves various subjective criteria and the weights of these criteria should be calculated based on a group of managers' opinions. The fuzzy set theory has the capability to deal with subjectivity and uncertainty existing in human preference. Therefore, fuzzy MCDM techniques generally enable us to integrate imprecise, vague, and incomplete data in our evaluation model systematically.

In this study, we integrate three MCDM methods by applying the modified Delphi, AHP and FTOPSIS methodologies. Considering the integrated approach, crisp values are used to assess the weights of all criteria and then linguistic values are used to determine the ratings of each alternative with respect to each criterion. In other words, the weights of evaluation criteria are determined through a modified Delphi and AHP methodology, and six sigma projects are then evaluated by applying FTOPSIS. Recently, integrated approaches have been extensively applied in the literature. Some examples of these applications include selection of the strategic alliance partner in logistic value chain (Büyüközkan et al., 2008), evaluation of Turkish cement firms' performance (Ertuğrul \& Karakaşoğlu, 2009), evaluation of hazardous waste transportation firms (Gumus, 2009), selection of the best mobile phone (Iş̧klar \& Büyüközkan, 2007), transshipment site selection (Önüt \& Soner, 2008), machine tool selection (Önüt et al., 2008), supplier selection (Önüt et al., 2009), evaluation of airline services quality (Tsaur et al., 2002) selection of the best fuel mode for public transportation (Tzeng et al., 2005), and selection of the most suitable bancassurance alliance structure $(\mathrm{Wu}$ et al., 2009). However, during the literature survey, we have not encountered an integrated decision-making method to guide managers in taking a proper decision about six sigma project selection. With the integrated application of the proposed method, evaluation process has advantages of the three methods and can never take into account the insufficiencies of each method. Therefore, the proposed fuzzy MCDM approach allows a more realistic tool for the project selection decision in a six sigma implementation process. The evaluation procedure of this study consists of three main steps:

Step 1: Use the modified Delphi method to define the criteria.

Step 2: Use the AHP method to calculate the criteria weights.

Step 3: Conduct FTOPSIS method to achieve the final ranking results.

\subsection{Modified Delphi Method}

Modified Delphi method replaces the conventionally Delphi method by simplifying the survey structure and summarizing the survey results. Modified Delphi method provides a structured communication process in which a group of experts input, discuss and defend their knowledge skills, expertise and opinions until a mutual consensus is achieved. The modified Delphi method consists of five steps (Chang et al., 2008): (1) select the experts; (2) conduct the first round of a survey; (3) conduct the second round of a questionnaire survey; (4) conduct the third round of a questionnaire survey; and (5) integrate a group of experts' opinions to reach a consensus. Steps (3) and (4) are typically repeated until a consensus is reached on a given topic.

AHP methodology depends on a group of experts' opinions. Therefore, criteria weights must be determined by experts who have a good understanding of evaluation criteria and their impact on the selection of an appropriate six sigma project. Murry and Hammons (1995) proposed that the Modified Delphi method must summarize the results of experts' opinions on a range from 10 to 30 . In this study, a group of experts consisting of 13 professionals from the case company participated in the decision-making group. To ensure non-interference, expert group opinions are accumulated and synthesized to identify the main factors for consideration in the evaluation criteria for six sigma project selection for the case company (Chang et al., 2008).

\section{2. $A H P$}

AHP is developed to solve complex MCDM problems involving multiple qualitative and quantitative criteria. 
It allows decision-makers to specify their preferences using the Saaty's 1-9 scale (Saaty, 1980). This scale can be very useful in helping a group of experts or an individual to make a decision. The purpose of AHP is to provide weights for each criterion and alternatives. AHP requires three steps: (i) identifying evaluation criteria, (ii) assessing the decision-maker evaluations by pairwise comparisons, and (iii) calculating the weights for criteria and alternatives. In AHP, logical consistency is also considered by evaluating the validity of the pairwise comparison process obtained from decisionmakers' preferences. The AHP procedure consists of the following steps (Chang et al., 2008; Işıklar \& Büyüközkan, 2007; Önüt \& Soner, 2008; Saaty, 1980; Wu et al., 2009):

Step 1: Establish a pairwise comparison decision matrix $(A)$

Let $C_{1}, C_{2}, \cdots C_{n}$ denote the set of elements, while $a_{i j}$ represents a quantified judgment on a pair of elements $C_{i}, C_{j}$. The relative importance of two elements is rated using a scale with the values $1,3,5,7$, and 9, where 1 refers to "equally important", 3 denotes "slightly more important", 5 equals "strongly more important", 7 represents "demonstrably more important" and 9 denotes "absolutely more important". Also, 2, 4, 6, and 8 are used for compromise between the above values. This yields an $n$-by-n comparison matrix $A$ as follows:

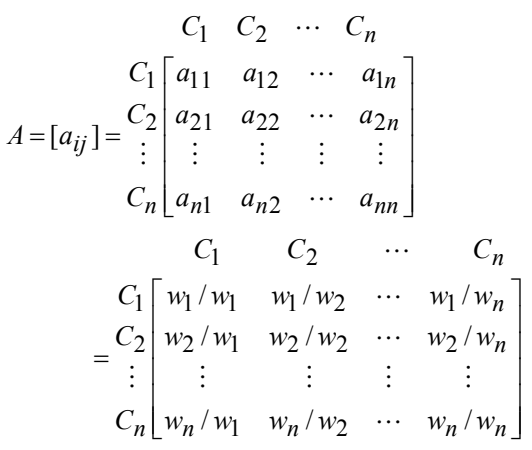

If matrix $A$ is consistent, the relations between weight $w_{i}$ and judgments $a_{i j}$ will satisfy the following conditions: $a_{i j}=w_{i} / w_{j}=1 / a_{j i}$ and $a_{i i}=1$ with $i, j=1,2, \cdots, n$.

Step 2: Normalize the decision matrix and calculate the priorities of this matrix

Before all the calculations of vector of priorities, the comparison matrix has to be normalized. For this purpose, each set of column values is summed. Then, each value is divided by the corresponding summed value. Finally, the average of rows is calculated and the relative weights of criteria $w_{1}, w_{2}, \cdots, w_{i}$ are obtained.

Step 3: Do consistency checks

The relative weights, which would also present the eigenvalues of criteria, should verify:

$A * w_{i}=\lambda_{\max } * w_{i}, \quad i=1,2, \cdots, n$.

Where $A$ represents the pairwise comparison decision matrix and $\lambda_{\max }$ gives the highest eigenvalue. Then consistency index (CI), which measures the inconsistencies of pairwise comparisons is calculated as:

$C I=\frac{\lambda_{\max }-n}{n-1}$

The last ratio that has to be calculated is consistency ratio (CR). The $\mathrm{CR}$ is computed by dividing the $\mathrm{CI}$ by a value obtained from a table of Random Index (RI):

$C R=\frac{C I}{R I}$

Generally, if CR is less than 0.10 , the comparisons are consistent and acceptable, otherwise not. RI denotes the average index for randomly generated weights. To check for consistency, the table of random indexes of the matrices of order 1 to 10 can be used as seen in Saaty (1980).

\subsection{The Fuzzy TOPSIS Method}

TOPSIS is a widely accepted multiple criteria method to identify solutions from a finite set of alternatives. Fuzzy TOPSIS (FTOPSIS) is first presented in Chen and Hwang (1992), with reference to Hwang and Yoon (1981). The basic principle of the fuzzy TOPSIS is that the chosen alternative should have the shortest distance from the positive ideal solution and the farthest distance from the negative-ideal solution in a geometrical (i.e., Euclidean) sense (Hwang \& Yoon, 1981).

It is often difficult for a decision-maker to assign a precise performance rating to an alternative for the criteria under consideration. The merit of using a fuzzy approach is to assign the relative importance of criteria using fuzzy numbers instead of crisp numbers (Yang 
and Hung, 2007). This section presents the proposed FTOPSIS methodology. In the following, some basic definitions of fuzzy sets are reviewed and summarized from Buckley (1985), Chen (2000), Kaufmann and Gupta (1985), Önüt and Soner (2008), Yang and Hung (2007), Zadeh (1965), and Zimmermann (1991).

Definition 1. A fuzzy number $\widetilde{a}$ in a universe of discourse $X$ is characterized by a membership function $\mu \widetilde{a}(x)$ which associates with each element $x$ in $X$, a real number in the interval $[0,1]$. The function value $\mu_{\widetilde{a}}(x)$ is termed the grade of membership of $x$ in $\tilde{a}$.

The present study uses triangular fuzzy numbers (TFNs). A TFN $\tilde{a}$ can be defined by a triplet $\left(a_{1}, a_{2}, a_{3}\right)$. Its conceptual schema and mathematical form are shown by Equation (6):

$\mu_{\widetilde{a}}(x)= \begin{cases}0, & x \leq a_{1}, \\ \frac{x-a_{1}}{a_{2}-a_{1}}, & a_{1}<x \leq a_{2}, \\ \frac{a_{3}-x}{a_{3}-a_{2}}, & a_{2}<x \leq a_{3}, \\ 0, & x>a_{3},\end{cases}$

Definition 2. Let $\widetilde{a}=\left(a_{1}, a_{2}, a_{3}\right)$ and $\widetilde{b}=\left(b_{1}, b_{2}, b_{3}\right)$ be two triangular fuzzy numbers, then the vertex method is defined to calculate the distance between them, as Equation (7):

$d(\widetilde{a}, \tilde{b})=\sqrt{\frac{1}{3}\left[\left(a_{1}-b_{1}\right)^{2}+\left(a_{2}-b_{2}\right)^{2}+\left(a_{3}-b_{3}\right)^{2}\right]}$

Also, the basic arithmetic operations on TFNs are as follows:

$$
\begin{aligned}
& \tilde{a}+\widetilde{b}=\left(a_{1}, a_{2}, a_{3}\right)(+)\left(b_{1}, b_{2}, b_{3}\right)=\left(a_{1}+b_{1}, a_{2}+b_{2}, a_{3}+b_{3}\right) \\
& \tilde{a}-\tilde{b}=\left(a_{1}, a_{2}, a_{3}\right)(-)\left(b_{1}, b_{2}, b_{3}\right)=\left(a_{1}-b_{1}, a_{2}-b_{2}, a_{3}-b_{3}\right) \\
& \tilde{a} \times \tilde{b} \cong\left(a_{1}, a_{2}, a_{3}\right)(\times)\left(b_{1}, b_{2}, b_{3}\right)=\left(a_{1} \times b_{1}, a_{2} \times b_{2}, a_{3} \times b_{3}\right) \\
& \tilde{a} \div \tilde{b} \cong\left(a_{1}, a_{2}, a_{3}\right)(\div)\left(b_{1}, b_{2}, b_{3}\right)=\left(a_{1} \div b_{3}, a_{2} \div b_{2}, a_{3} \div b_{1}\right) \\
& (\widetilde{a})^{-1}=\left(a_{1}, a_{2}, a_{3}\right)^{-1}=\left(1 / a_{3}, 1 / a_{2}, 1 / a_{1}\right) \\
& k \widetilde{a}=k \times\left(a_{1}, a_{2}, a_{3}\right)=\left(k a_{1}, k a_{2}, k a_{3}\right)
\end{aligned}
$$

The terms used in this study are briefly described as follows: (a) Alternatives: A set of possible $m$ alternatives $A_{i}$, for $i=1,2, \cdots, m$,

(b) Criteria: $\mathrm{A}$ set of possible $n$ criteria $C_{j}$ for $j=1,2, \cdots, n$,

(c) Criterion weights: A set of relative importance of each criterion $\widetilde{w}_{j}$ for $j=1,2, \ldots, n$,

(d) Alternative scores: A set of performance ratings of alternatives $A_{i}(i=1,2, \cdots, m)$ with respect to criteria $C_{j}$ $(j=1,2, \cdots, n)$ called $\tilde{x}_{i j}(i=1,2, \ldots, m, j=1,2, \ldots, n)$.

Then, the problem can be concisely expressed in matrix format as Equations (14) and (15):

$$
\begin{aligned}
& \begin{array}{llllll}
C_{1} & C_{2} & \ldots & C_{j} & \ldots & C_{n}
\end{array} \\
& A_{1}\left[\begin{array}{llllll}
\tilde{x}_{11} & \widetilde{x}_{12} & \ldots & \tilde{x}_{1 j} & \ldots & \tilde{x}_{1 n}
\end{array}\right] \\
& \begin{array}{c|cccccc}
A_{2} & \tilde{x}_{21} & \tilde{x}_{22} & \ldots & \tilde{x}_{2 j} & \ldots & \tilde{x}_{2 n}
\end{array} \\
& \widetilde{D}=\begin{array}{c|cccccc}
\vdots & \vdots & \vdots & \vdots & \vdots & \vdots & \vdots \\
A_{i} & \tilde{x}_{i 1} & \tilde{x}_{i 2} & \ldots & \tilde{x}_{i j} & \ldots & \tilde{x}_{i n}
\end{array} \\
& A_{m}\left[\begin{array}{cccccc}
\vdots & \vdots & \vdots & \vdots & \vdots & \vdots \\
\tilde{x}_{m 1} & \tilde{x}_{m 2} & \ldots & \tilde{x}_{m j} & \ldots & \tilde{x}_{m n}
\end{array}\right] \\
& \widetilde{W}=\left[\widetilde{w}_{1}, \widetilde{w}_{2}, \ldots \widetilde{w}_{j}\right],
\end{aligned}
$$

Where $\quad \tilde{x}_{i j}=\left(a_{i j}, b_{i j}, c_{i j}\right)$ and $\quad \tilde{w}_{j}=\left(w_{j 1}, w_{j 2}, w_{j 3}\right) \quad$ are TFNs (for $i=1,2, \cdots, m, j=1,2, \cdots, n$ ). Note that $\tilde{x}_{i j}$ represents the performance rating of the $i$ th alternative, $A_{i}$, with respect to $j$ th criterion $C_{j}$, and $\widetilde{w}_{j}$ represents the weight of the $j$ th criterion $C_{j}$.

The normalized fuzzy decision matrix denoted by $\widetilde{R}$ is shown as Equation (16):

$$
\widetilde{R}=\left[\widetilde{r}_{i j}\right]_{m \times n} \quad i=1,2,3, \cdots, m, \quad j=1,2,3, \cdots, n .
$$

The weighted normalized fuzzy decision matrix is constructed as Equation (17):

$$
\widetilde{V}=\left[\begin{array}{cccccc}
\widetilde{v}_{11} & \widetilde{v}_{12} & \ldots & \widetilde{v}_{1 j} & \ldots & \widetilde{v}_{1 n} \\
\vdots & \vdots & \vdots & \vdots & \vdots & \vdots \\
\widetilde{v}_{i 1} & \widetilde{v}_{i 2} & \ldots & \widetilde{v}_{i j} & \ldots & \widetilde{v}_{i n} \\
\vdots & \vdots & \vdots & \vdots & \vdots & \vdots \\
\widetilde{v}_{m 1} & \widetilde{v}_{m 2} & \ldots & \widetilde{v}_{m j} & \ldots & \widetilde{v}_{m n}
\end{array}\right]
$$

$$
=\left[\begin{array}{cccccc}
\widetilde{w}_{1} \widetilde{r}_{11} & \widetilde{w}_{2} \widetilde{r}_{12} & \ldots & \widetilde{w}_{j} \widetilde{r}_{1 j} & \ldots & \widetilde{w}_{n} \widetilde{r}_{1 n} \\
\vdots & \vdots & \vdots & \vdots & \vdots & \vdots \\
\widetilde{w}_{1} \widetilde{r}_{i 1} & \widetilde{w}_{2} \widetilde{r}_{i 2} & \ldots & \widetilde{w}_{j} \widetilde{r}_{i j} & \ldots & \widetilde{w}_{n} \widetilde{r}_{i n} \\
\vdots & \vdots & \vdots & \vdots & \vdots & \vdots \\
\widetilde{w}_{1} \widetilde{r}_{m 1} & \widetilde{w}_{2} \widetilde{r}_{m 2} & \ldots & \widetilde{w}_{j} \widetilde{r}_{m j} & \ldots & \widetilde{w}_{n} \widetilde{r}_{m n}
\end{array}\right]
$$

Given the above equations of the fuzzy set theory, the main steps of the FTOPSIS algorithm can be defined as follows: 
Step 1: Choose the linguistic ratings $\left(\tilde{x}_{i j}, i=1,2, \ldots, m, j=1,2, \ldots, n\right)$ for alternatives with respect to criteria. The fuzzy linguistic rating $\left(\tilde{x}_{i j}\right)$ preserves the property that the ranges of TFNs belong to $[0,1]$; thus, there is no need for normalization. In this situation, the $\widetilde{D}$ defined by Equation (14) is equivalent to the $\widetilde{R}$ defined by Equation (16).

Step 2: Calculate the weighted normalized fuzzy decision matrix. The weighted fuzzy normalized value $\widetilde{V}$ is calculated by Equation (17).

Step 3: Identify the positive ideal $\left(A^{*}\right)$ and negative ideal $\left(A^{-}\right)$solutions. The fuzzy positive-ideal solution (FPIS, $A^{*}$ ) and fuzzy negative ideal solution (FNIS, $A^{-}$) are shown as Equations (18) and (19):

$A^{*}=\left\{v_{1}^{*}, v_{2}^{*} \cdots, v_{n}^{*}\right\}=\left\{\left(\max _{i} v_{i j} \mid j \in J_{1}\right),\left(\min _{i} v_{i j} \mid j \in J_{2}\right), \quad i=1,2, \ldots, m\right\}$

$$
A^{-}=\left\{v_{1}^{-}, v_{2}^{-} \cdots, v_{n}^{-}\right\}=\left\{\left(\min _{i} v_{i j} \mid j \in J_{1}\right),\left(\max _{i} v_{i j} \mid j \in J_{2}\right), i=1,2, \ldots, m\right\}
$$

where $J_{1}$ is a set of benefit criteria, and $J_{2}$ is a set of cost criteria.

Step 4: Calculate separation measures. The distances of each alternative from $A^{*}$ and $A^{-}$can be calculated using Equations (20) and (21):

$$
\begin{aligned}
& d_{i}^{*}=\sum_{j=1}^{n} d\left(\tilde{v}_{i j}, \tilde{v}_{j}^{*}\right), \quad i=1,2, \ldots, m \\
& d_{i}^{-}=\sum_{j=1}^{n} d\left(\tilde{v}_{i j}, \tilde{v}_{j}^{-}\right), \quad i=1,2, \ldots, m
\end{aligned}
$$

Step 5: Calculate similarities to ideal solution. This is calculated by Equation (22):

$C C_{i}=\frac{d_{i}^{-}}{d_{i}^{*}+d_{i}^{-}}$

Step 6: Rank preference order. Choose an alternative with maximum $C C_{i}$ or rank alternatives according to $C C_{i}$ in descending order.

\section{The Application of Proposed Framework to Six Sigma Project Selection}

The problem is the evaluation of the potential six sigma projects and selection of the best one. For this purpose, first of all, the evaluation criteria are obtained with the consultation of the case company managers by using the Modified Delphi method. Secondly, by applying the AHP methodology given in Section 2.2, the importance weights of the evaluation criteria are calculated. These weights are then used as FTOPSIS inputs. Finally, FTOPSIS is operated for the evaluation problem and the final ranking of the preference order for the potential five Six Sigma projects are gained. At the end of this section, the results of the proposed methodology and the changes in the weights of different main criteria are analyzed in detail by a sensitivity analysis.

\subsection{Brief Information about the Turkish Automotive Supplier Industry and the Case Company}

The Turkish automotive industry is viewed as locomotive, largest exporter, leading investor, and economically strategic sector of the Turkish economy. According to Automotive Manufacturers Association (OSD) seventeen OEM (Original Equipment Manufacturers), members of OSD, operate in Turkey. The Turkish automotive sector has reached an export value of $\$ 21,889$ billion in 2008 , and it projects that this figure will increase to $\$ 25$ billion by the end of 2009 (OSD, 2008). This figure puts the sector in the top position in the sectors that export to other countries in Turkey. In parallel to the development of the OEM industry, the supplier industry has also developed in Turkey. The number of supplier companies in Turkey is around 900 and 226 of them are members of the Association of Automotive Parts and Components Manufacturers (TAYSAD). These companies manufacture original equipments for the OEM industry directly and they also export to other markets. These companies also represent $65 \%$ of the output of the Turkish automotive supplier industry and $70 \%$ of the industry's exports. Also, exports of the Turkish automotive supplier industry have increased to $\$ 7.016$ billion in 2008 (OSD, 2008), and it projects that it will have reached to $\$ 8$ billion by the end of 2009 . Recently, due to the high export potential and Turkey's regional advantages, foreign company partnerships have been showing an increasing interest in the automotive 
supplier industry in Turkey. Thus, 185 global suppliers such as Autoliv, Bosch, Delphi, Valeo, and Denso have made important investments due to the fact that Turkey provide cheap and qualified labor force, with high standards of production. These companies have preferred especially to form joint ventures with the local supplier firms.

The company providing the context for this application is a stand-alone division of a multinational corporation. It was established as a joint venture company in 1997 in Istanbul, Turkey. Between the years 1997-2009, it has continued growth both in investment and market share. The company now has more than 950 employees and has achieved production-based sales of approximately 400 million dollars for the year ending 2008. The main customers of the company are Toyota, Renault, Ford, Tofas-Fiat, and Hyundai/Kia. The company currently produces airbags, seatbelts, steering wheels, and safety electronics. Also, the company maintains an advanced TQM process, ISO 9000 quality systems (ISO 9000, QS 9000, ISO 9002, ISO/TS 16949, ISO 14001 , etc.) and process audit. However, the management of the company realized that the TQM process and its associated systems were too slow in responding to customer requirements and it was only realized by applying appropriate six sigma projects. For this purpose they discussed how to establish an organizational infrastructure for six sigma implementation by highlighting the significance of top management commitment, training programs, customer requirements, and a system of quality experts (ie., Black Belts, Green Belts, and so on). According to them, the key ingredient for the successful Six Sigma implementation is project prioritization and selection.

Also the company desires to create a low cost advantage by improving CTQ (critical to quality) characteristics and performance issues, and making other process changes in response to customer needs and expectations. To achieve these objectives, company's managers need to select suitable six sigma projects. Six sigma projects will also provide better competitive advantage. In line with this purpose, they believe that the firm's six sigma strategy when combined with the organization's goals and expectations will also result in success of six sigma implementation. In here, after preliminary screening, five six sigma project alternatives remain for further evaluation. Thus, the company managers are now faced with the challenges of efficiently evaluating five six sigma project proposals. For confidentiality, we will name those projects as A1, A2, A3, A4, and A5. For handling this MCDM problem of six sigma project selection, the case company adopted our proposed method and set up a decision-making group consisting of 13 professionals such as, general manager, and several managers representing the marketing, financial, production, human resource, and information technology department. All experts identified the importance of six sigma projects for the company's future development. The following shows how the case company utilized our proposed method to evaluate and select the best six sigma project logically.

\subsection{The Application of Proposed Method}

The main steps of the proposed evaluation framework aimed at selecting the best six sigma project for the company are as follows:

Step 1: Define the criteria to evaluate six sigma project alternatives with the modified Delphi method.

In the first step, 13 experts from the case company participated in the modified Delphi-based decisionmaking group to identify evaluation criteria. Then, six sigma project selection criteria were determined based on the detailed review of literature (mainly based on the studies of Antony, Kumar, \& Madu, 2005; Antony, 2004; Banuelas et al., 2006; Coronado \& Antony, 2002; Eckes, 2000; Henderson \& Evans, 2000; Kumar et al., 2007; Pande et al., 2000; Pyzdek, 2000) and face-toface interviews undertaken with 13 experts from relevant departments of the case company. After a general consensus among experts, five evaluation criteria and five six sigma project alternatives (A1, A2, $\mathrm{A} 3, \mathrm{~A} 4$, and A5) for the implementation of six sigma projects were identified through the related references and experts' statements. The criteria involved in this study include: financial benefits, quality, process capability, customer satisfaction, and operational costs.

Step 2: Establish pairwise comparison matrix and calculate the weights of this matrix by AHP

In this step, all the relative scores of the criteria, obtained from the pairwise comparisons of the 13 
experts, have been aggregated using the geometric mean method. We used the Equations (1) and (2) to calculate the aggregate pairwise comparison matrix. Table 1 summarizes aggregate pairwise comparison matrix for the criteria used in the study. The comparison matrix of the criteria in Table 1 is then used to calculate the weights of this matrix using Equation (3). Furthermore, we used Equations (4) and (5) to perform the consistency checks (Wu et al., 2009). $C I$ and $C R$ of the aggregate pairwise comparison matrix provided by the 13 experts are below 0.10 , indicating a satisfactory degree of consistency (Saaty, 1980). Therefore, AHP calculations are consistent. Otherwise, those experts may need to reconsider their evaluations.
By using Table 2, linguistic variables are transformed to fuzzy preferences. For example, the fuzzy variableVery low has its associated triangular fuzzy number with minimum of 0.00 , mode of 0.00 , and maximum of 0.10 . The same definition is then applied to the other fuzzy variables-Low, Medium low (ML), Medium (M), Medium high $(\mathrm{MH})$, High $(\mathrm{H})$, and Very high $(\mathrm{VH})$ (Yang \& Hung, 2007). Thus, the fuzzy decision matrix representing fuzzy preferences for the five candidate projects are shown in Table 3. This is the third step of the FTOPSIS analysis.

Table 1. Pairwise comparisons of selection criteria

\begin{tabular}{ccccccccc}
\hline & $\mathrm{C} 1$ & $\mathrm{C} 2$ & $\mathrm{C} 3$ & $\mathrm{C} 4$ & $\mathrm{C} 5$ & $w i$ & $A^{*} w i$ & $A^{*} w i / w i$ \\
\hline $\mathrm{C} 1$ & 1 & 3 & 7 & 0,333 & 9 & 0,276 & 1,523 & 5,521 \\
$\mathrm{C} 2$ & 0,333 & 1 & 3 & 0,2 & 7 & 0,136 & 0,713 & 5,231 \\
$\mathrm{C} 3$ & 0,143 & 0,3333 & 1 & 0,111 & 3 & 0,056 & 0,289 & 5,119 \\
$\mathrm{C} 4$ & 3 & 5 & 9 & 1 & 9 & 0,501 & 2,794 & 5,578 \\
$\mathrm{C} 5$ & 0,111 & 0,1429 & 0,3333 & 0,111 & 1 & 0,031 & 0,155 & 5,059 \\
& & & & & & & $\mathrm{CI}=0.075$ & $\mathrm{CR}=0.068$ \\
\hline
\end{tabular}

Step 3: Transform linguistic variables to fuzzy preferences

In this step, we use the linguistic variables to evaluate the ratings of alternatives with respect to various criteria. The decision makers use the linguistic variables shown in Table 2 to assess the ratings of alternatives with respect to each criterion. Among the commonly used fuzzy numbers, triangular fuzzy numbers (TFNs) are likely to be the most appropriate ones due to their simplicity in modeling and ease of computation (Yang \& Hung, 2007). In this study, the seven-level fuzzy linguistic variables that can be expressed in positive TFNs (Chen, 2000) are used shown in Table 2.

Table 2. Linguistic scale for the ratings of each alternative

\begin{tabular}{lc}
\hline Linguistic variables & Fuzzy preference \\
\hline Very low (VL) & $(0,0,0.1)$ \\
Low (L) & $(0,0.1,0.3)$ \\
Medium low (ML) & $(0.1,0.3,0.5)$ \\
Medium (M) & $(0.3,0.5,0.7)$ \\
Medium high (MH) & $(0.5,0.7,0.9)$ \\
High (H) & $(0.7,0.9,1.0)$ \\
Very high (VH) & $(0.9,1.0,1.0)$ \\
\hline
\end{tabular}

Table 3. Fuzzy preferences for the five candidate projects

\begin{tabular}{|c|c|c|c|}
\hline & $\mathrm{C} 1$ & $\mathrm{C} 2$ & $\mathrm{C} 3$ \\
\hline A1 & $(0.9,1.0,1.0)$ & $(0.7,0.9,1.0)$ & $(0.3,0.5,0.7)$ \\
\hline A2 & $(0.5,0.7,0.9)$ & $(0.5,0.7,0.9)$ & $(0.7,0.9,1.0)$ \\
\hline A3 & $(0.3,0.5,0.7)$ & $(0.9,1.0,1.0)$ & $(0.5,0.7,0.9)$ \\
\hline A4 & $(0.7,0.9,1.0)$ & $(0.5,0.7,0.9)$ & $(0.9,1.0,1.0)$ \\
\hline \multirow[t]{2}{*}{ A5 } & $(0.5,0.7,0.9)$ & $(0.7,0.9,1.0)$ & $(0.1,0.3,0.5)$ \\
\hline & $\mathrm{C} 4$ & $\mathrm{C} 5$ & \\
\hline Al & $(0.7,0.9,1.0)$ & $(0.5,0.7,0.9)$ & \\
\hline A 2 & $(0.1,0.3,0.5)$ & $(0.7,0.9,1.0)$ & \\
\hline A3 & $(0.0,0.1,0.3)$ & $(0.3,0.5,0.7)$ & \\
\hline A4 & $(0.3,0.5,0.7)$ & $(0.3,0.5,0.7)$ & \\
\hline A5 & $(0.5,0.7,0.9)$ & $(0.0,0.1,0.3)$ & \\
\hline
\end{tabular}

Step 4: Calculate the fuzzy weighted decision matrix

The fourth step in the analysis is to find the fuzzy weighted decision matrix. After deriving the fuzzy preferences, fuzzy weighted decision matrix can be calculated by using Equation (17). The decision matrix must first be normalized so that all fuzzy numbers range within the interval $[0,1]$. However, the normalized decision matrix is the same as the fuzzy decision matrix because of the scale used for linguistic variables. Table 4 shows fuzzy weighted decision matrix. 
Table 4. Fuzzy weighted decision matrix

\begin{tabular}{cccccc}
\hline & $\mathrm{C} 1$ & $\mathrm{C} 2$ & $\mathrm{C} 3$ & $\mathrm{C} 4$ & $\mathrm{C} 5$ \\
\hline A1 & $(0.248,0.276,0.276)$ & $(0.095,0.123,0.136)$ & $(0.017,0.028,0.040)$ & $(0.351,0.451,0.501)$ & $(0.015,0.021,0.028)$ \\
A2 & $(0.138,0.193,0.248)$ & $(0.068,0.095,0.123)$ & $(0.040,0.051,0.056)$ & $(0.050,0.150,0.250)$ & $(0.021,0.028,0.031)$ \\
A3 & $(0.083,0.138,0.193)$ & $(0.123,0.136,0.136)$ & $(0.028,0.040,0.051)$ & $(0.000,0.050,0.150)$ & $(0.009,0.015,0.021)$ \\
A4 & $(0.193,0.248,0.276)$ & $(0.068,0.095,0.123)$ & $(0.051,0.056,0.056)$ & $(0.150,0.250,0.351)$ & $(0.009,0.015,0.021)$ \\
A5 & $(0.138,0.193,0.248)$ & $(0.095,0.123,0.136)$ & $(0.006,0.017,0.028)$ & $(0.250,0.351,0.451)$ & $(0.000,0.003,0.009)$ \\
\hline
\end{tabular}

Step 5: Determine the positive ideal and negative ideal solutions and calculate the separation measures

The fuzzy positive-ideal solution (FPIS, $A^{*}$ ) and fuzzy negative-ideal solution (FNIS, $A^{-}$) can be defined as: $\widetilde{v}_{j}^{*}=(1,1,1)$ and $\widetilde{v}_{j}^{-}=(0,0,0)$, for benefit criterion, and $\widetilde{v}_{j}^{*}=(0,0,0)$ and $\widetilde{v}_{j}^{-}=(1,1,1)$, for cost criterion. In this study, only the "operational cost" criterion is evaluated as a cost criterion and the others are considered as benefit criteria. The positive ideal $\left(\mathrm{A}^{*}\right)$ and negative ideal $\left(\mathrm{A}^{-}\right)$solutions is computed by using the Equations (18) and (19). The distances of each alternative from the positive ideal $\left(\mathrm{A}^{*}\right)$ and negative ideal $\left(\mathrm{A}^{-}\right)$solutions are then calculated by using Equations (20) and (21) in the fifth step of the analysis (Yang \& Hung, 2007).

Step 6: Calculate similarities to ideal solution and rank the preference order

Next, the similarities to an ideal solution are computed by using Equation (22) in the sixth step of the analysis. As the last step, the results of an integrated AHP and FTOPSIS methodology are summarized in Table 5. Based on $C C_{j}$ values, the ranking of the alternatives in descending order are A1, A5, A4, A2, and A3. Thus, the best alternative for the managers of the case company is selected as A1.

Table 5. Fuzzy TOPSIS results

\begin{tabular}{cccc}
\hline & $D_{j}^{*}$ & $D_{j}^{-}$ & $C C_{j}$ \\
\hline A1 & 3,179 & 1,833 & 0,366 \\
A2 & 3,545 & 1,490 & 0,296 \\
A3 & 3,644 & 1,394 & 0,277 \\
A4 & 3,382 & 1,642 & 0,327 \\
A5 & 3,334 & 1,693 & 0,337 \\
\hline
\end{tabular}

\subsection{Sensitivity Analysis}

The purpose of the sensitivity analysis is to demonstrate the influence of different criteria weights on Six Sigma project selection. For this reason, we have exchanged each criterion's weight with another criterion's weight, and hence ten combinations of the five criteria are analyzed, with each combination stated as a condition. For each condition, similarities to the ideal solution $C C_{j}$ are computed (Gumus, 2009; Önüt \& Soner, 2008). The results of the sensitivity analysis are summarized in Table 6 and graphical representation of these results is illustrated in Figure 1.

As can be seen from Table 6, the main condition expresses the original results of the integrated AHP and FTOPSIS methodology. Also, A1 has the highest $C C_{j}$ value of 0.370 when the first and fourth criteria weights are exchanged in condition 3. Also, A1 has the lowest value of 0.219 when the fourth and fifth criteria weights are exchanged in condition 10. A2 will have the highest $C C_{j}$ value of 0.344 when the third and fourth criteria weights are exchanged in condition 8 . A2 will have the lowest value of 0.184 when the fourth and fifth criteria weights are exchanged in condition 10. A3 will have the highest $C C_{j}$ value of 0.335 when the second and fourth criteria weights are exchanged in condition 6 . A3 will have the lowest value of 0.214 when the fourth and fifth criteria weights are exchanged in condition 10. A4 will have the highest $C C_{j}$ value of 0.367 when the third and fourth criteria weights are exchanged in condition 8. A4 will have the lowest value of 0.232 when the fourth and fifth criteria weights are exchanged in condition 10. A5 will have the highest $C C_{j}$ value of 0.349 when the second and fourth criteria weights are exchanged in condition 6. A5 will have the lowest value of 0.257 when the fourth and fifth criteria weights are exchanged in condition 10. As can be seen from Table 6, A1 and A4 are the most sensitive alternatives to changes in the weights of main criteria. In other words, A1 will be selected if conditions $1,2,3,5,6,7$, and 9 are met. On the other hand, A4 will be selected in condition 8 and A5 will be selected in conditions 4 and 10. Also, Figure 1 shows that the superiority of A1 decreases in conditions 4,8 , and 10 . So, it is clear that the weights of main criteria are very influential to the selection process. These results highlight the importance of sensitivity analysis for various criteria weights. 
Table 6. Results of the sensitivity analysis

\begin{tabular}{|c|c|c|c|c|c|c|c|c|c|c|}
\hline \multirow[t]{2}{*}{ Conditions } & \multicolumn{5}{|c|}{ Weights } & \multicolumn{5}{|l|}{$C C_{j}$} \\
\hline & $\mathrm{w}_{1}$ & $\mathrm{w}_{2}$ & $\mathrm{w}_{3}$ & $\mathrm{w}_{4}$ & $\mathrm{~W}_{5}$ & A1 & $\mathrm{A} 2$ & A3 & A4 & A5 \\
\hline Main & 0.276 & 0.136 & 0.056 & 0.501 & 0.031 & 0.366 & 0.296 & 0.277 & 0.327 & 0.337 \\
\hline 1 & 0.136 & 0.276 & 0.056 & 0.501 & 0.031 & 0.363 & 0.296 & 0.289 & 0.322 & 0.341 \\
\hline 2 & 0.056 & 0.136 & 0.276 & 0.501 & 0.031 & 0.346 & 0.303 & 0.285 & 0.331 & 0.320 \\
\hline 3 & 0.501 & 0.136 & 0.056 & 0.276 & 0.031 & 0.370 & 0.313 & 0.292 & 0.343 & 0.337 \\
\hline 4 & 0.031 & 0.136 & 0.056 & 0.501 & 0.276 & 0.284 & 0.219 & 0.227 & 0.260 & 0.295 \\
\hline 5 & 0.276 & 0.056 & 0.136 & 0.501 & 0.031 & 0.360 & 0.298 & 0.273 & 0.331 & 0.328 \\
\hline 6 & 0.276 & 0.501 & 0.056 & 0.136 & 0.031 & 0.366 & 0.324 & 0.335 & 0.341 & 0.349 \\
\hline 7 & 0.276 & 0.031 & 0.056 & 0.501 & 0.136 & 0.333 & 0.263 & 0.246 & 0.301 & 0.315 \\
\hline 8 & 0.276 & 0.136 & 0.501 & 0.056 & 0.031 & 0.334 & 0.344 & 0.325 & 0.367 & 0.303 \\
\hline 9 & 0.276 & 0.136 & 0.031 & 0.501 & 0.056 & 0.359 & 0.287 & 0.270 & 0.319 & 0.334 \\
\hline 10 & 0.276 & 0.136 & 0.056 & 0.031 & 0.501 & 0.219 & 0.184 & 0.214 & 0.232 & 0.257 \\
\hline
\end{tabular}

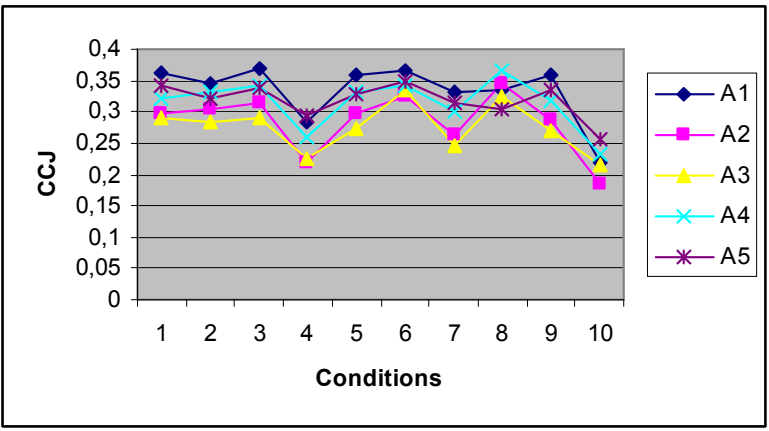

Fig. 1. Sensitivity analysis

\section{Discussion and limitations}

In recent years, it appears that a particular emphasis has been placed by researchers on the problems of six sigma project selections. However, there still lacks a well-structured approach to assist a company in selecting the most suitable project. Thus, the objective of this study is to analyze the potential six sigma projects, and to choose the best one among them by using an integrated fuzzy MCDM approach. To show the potential of the methodology, an actual case application was presented. Sensitivity analysis was also performed to investigate the sensitivity of the results to changes in the weights of main criteria. Thus, this study aimed at searching an improved solution to the six sigma project selection problem. This is the most powerful motivation to consider this problem. For this purpose, this paper prioritizes and then ranks the five six sigma projects by applying the modified Delphi, AHP, and FTOPSIS methodologies. Saaty (1994) claimed that AHP has some advantages. First, it helps to decompose an unstructured problem into a reliable hierarchic structure that includes various criteria and alternatives to determine the best choice. Second, it can elicit judgments from decision-makers to determine weights of the elements. Third, it uses an admissible consistency ratio to validate the consistency of the decision-making process. Besides, we applied FTOPSIS method to complement AHP method for this kind of real-life problem. FTOPSIS is rational, understandable and easily programmable computation procedure. It can effectively deal with imprecision or vagueness existed in human preference. Also, it has the capability of representing vague data. Therefore, fuzzy MCDM methodology is a preferred choice in solving the proposed six sigma evaluation problem since the scores of the five candidate projects are imprecise or vague. With the integrated application of the modified Delphi, AHP, and FTOPSIS methods, evaluation process has the advantages of all these methods.

Although the proposed model provides a comprehensive framework to guide the managers of the case company, there are some limitations of the model. First, the proposed method did not consider all possible criteria that could be added to the model. The criteria for six sigma project selection may include cycle time, capacity, internal performance, increase in productivity, cost of poor quality, defects rate, supplier quality etc. Therefore, the weightings of the criteria obtained by using the AHP may change if a new criterion is added to the model. Besides, the additional factors require the additional time and effort necessary for completion of such a model. In this case, the formation of the pairwise 
comparison matrices would be a time-consuming and complex task. Second, the inputs of the AHP are mainly collected from the company's experts. So, the model is very dependent on the weightings provided by the experts' opinions. On the other hand, the proposed method has been effectively used in the decision of six sigma project evaluation problem. In fact, the management of the case company adopted our proposed framework to logically deal with that complicated evaluation problem and selected A1 as the best six sigma project. Managers can use this result in managing, benchmarking, and continuously improving their project selection decisions. Moreover, the integrated method learned from the study can be useful to the case company's future evaluation problems.

\section{Conclusions}

Six sigma projects have a vital role in today's organizations to realize their vision and strategies. Companies implement six sigma projects to improve quality, reduce variation, and eliminate waste of their manufacturing systems. For this purpose, the practical application from an automotive supplier company was adopted for empirical analysis. Therefore, the major contribution of this research lies in the development of a comprehensive fuzzy MCDM model, which incorporates various factors for selecting the best six sigma project for the case company.

Use of an integrated fuzzy MCDM methodology offers a number of benefits. Firstly, it is a systematic and reliable method since it is capable of capturing an expert's opinions when complex multi-criteria decisionmaking problems are considered. AHP model is developed to take both quantitative and qualitative criteria with crisp values into account. Then FTOPSIS is preferred to handle imprecise or vague performance scores used for the five project alternatives. Thus, the use of AHP weights in FTOPSIS makes the evaluation process more rational and realistic. Because of this ability, managers can use this method in making their strategic decisions. Secondly, it considers the hierarchical structure, pairwise comparisons and consistency checks in the decision process. Therefore, it can be said that it is an excellent tool to handle quantitative and qualitative assessments about six sigma project evaluation problem, and its calculations are faster than other analytical techniques such as simple project assessment techniques, statistical and mathematical programming approaches. Finally, the combined modified Delphi, AHP, and FTOPSIS methodologies are very flexible and suitable for various decision situations. They have been effectively used in the decision of six sigma project evaluation problem. In addition, the proposed framework, with minor modifications, can be useful to all firms in their project evaluation decisions. It is anticipated that a further study will improve the method to deal with other management problems. Future work includes applying the fuzzy MCDM methods to different companies operating in various industries with some specific changes. In addition, comparing this method with different fuzzybased models may be a further research area.

\section{References}

1. A. Kaufmann and M.M. Gupta, Introduction to Fuzzy Arithmetic: Theory and Applications, (Von Nostrand Reinhold, New York, 1985).

2. A.T. Gumus, Evaluation of Hazardous Waste Transportation Firms by Using a Two Step Fuzzy-AHP and TOPSIS Methodology, Expert Syst Appl. 36(2) (2009) 4067-4074.

3. C. Adams, P. Gupta and C. Wilson, Six Sigma Deployment (Butterworth-Heinemann, Oxford, 2003).

4. C. Kahraman and A.C. Tolga, An Alternative Ranking Approach and Its Usage in Multi-Criteria DecisionMaking, Int J Comput Intelligence Syst. 2(3) (2009) 219235.

5. C.A. Hendricks and R.L. Kelbaugh, Implementing Six Sigma at GE, J for Qual and Partici. 21(4) (1998) 48-53.

6. C.L. Hwang and K. Yoon, Multiple Attribute Decision Making Methods and Applications (New York: Springer, (1981).

7. C.R. Wu, C.T. Lin and Y.F. Lin, Selecting the Preferable Bancassurance Alliance Strategic By Using Expert Group Decision Technique, Expert Syst Appl. 36(2) (2009) 3623-3629.

8. C.T. Chen, Extensions of the TOPSIS for group Decision Making under Fuzzy Environment, Fuzzy Set Syst. 114(1) (2000) 1-9.

9. C.T. Su and C.J. Chou, A systematic methodology for the creation of Six Sigma projects: A case study of semiconductor foundry, Expert Syst Appl. 34(4) (2008) 2693-2703.

10. C.W. Chang, C.R. Wu and H.C. Chen, Using Expert Technology to Select Unstable Slicing Machine to Control Wafer Slicing Quality Via Fuzzy AHP, Expert Syst Appl. 34 (2008) 2210-2220.

11. F. Breyfogle, J. Cupello and B. Meadws, Managing Six Sigma, (Wiley Inter-Science, New York, 2001). 
12. G. Büyüközkan, O. Feyzioğlu and E. Nebol, Selection of the Strategic Alliance Partner in Logistics Value Chain, Int J Prod Econ. 113(1) (2008) 148-158.

13. G. Eckes, The Six Sigma Revolution (Wiley InterScience, New York, 2000).

14. G. Işıklar and G. Büyüközkan, Using A Multi-Criteria Decision Making Approach to Evaluate Mobile Phone Alternatives, Comp Stand Inter. 29(2) (2007) 265-274.

15. G.H. Tzeng, C.W. Lin and S. Opricovic, Multi-Criteria Analysis of Alternative-Fuel Buses for Public Transportation, Energ Policy, 33(11) (2005) 1373-1383.

16. G.J. Hahn, N. Doganaksoy and R. Hoerl, The Evolution of Six-Sigma, Qual Eng. 12(3) (2000)317-326.

17. G.J. Hahn, W.J. Hill, R.W. Hoerl and S.A. Zinkgraf, The Impact of Six Sigma Improvement-A Glimpse into the Future of Statistics, Am Stat. 53(3) (1999) 208-215.

18. H.J. Zimmermann, Fuzzy Set Theory and Its Applications, 2nd edn. (Kluwer Academic Publishers, London, 1991).

19. I. Ertuğrul and N. Karakaşoğlu, Performance Evaluation of Turkish Cement Firms with Fuzzy Analytic Hierarchy Process and TOPSIS Methods, Expert Syst Appl. 36(1) (2009), 702-715.

20. J. Antony, M. Kumar, C.N. Madu, Six Sigma in SmallAnd Medium-Sized UK Manufacturing Enterprises: Some Empirical Observations, Int J Qual Reliab Manage. 22(8) (2005) 860-874.

21. J. Antony, Six Sigma in the UK Service Organizations: Results from A Pilot Survey, Managerial Auditing $J$. 19(8), (2004) 1006-1013.

22. J. Motwani, A. Kumar and J. Antony, A business process change framework for examining the implementation of Six Sigma: a case study of Dow chemicals, The TQM Mag. 16(4) (2004) 273-283

23. J.J. Buckley, Fuzzy Hierarchical Analysis, Fuzzy Set Syst. 17(3) (1985) 233-247.

24. J.P. Zimmerman, and J. Weiss, Six Sigma's Seven Deadly Sins, Quality, 44(1) (2005) 62-67.

25. J.W. Murry, and J.O. Hammons, Delphi: A Versatile Methodology for Conducting Qualitative Research, Rev High Educ. 18(4) (1995) 423-436.

26. K. Linderman, R.G. Schroeder, S. Zaheer, A.S. Choo, Six Sigma: A Goal-Theoretic Perspective, J Oper Manag. 21(2) (2003) 193-203.

27. K.M. Henderson and J.R. Evans, Successful Implementation Of Six Sigma: Benchmarking General Electric Company, Benchmarking: An Int J. 7(4) (2000) 260-281

28. L.A. Zadeh, Fuzzy sets, Inform Control. 8 (1965) 338353.

29. M. Kelly, Three steps to project selection, ASQ Six Sigma Forum Mag. 2(1) (2002) 29-33.

30. OSD (2008) Available at: http://www.osd.org.tr/2008rapor (Accessed May 2009)

31. P.S. Pande, R.P. Neuman, and R.R. Cavanagh, The Six Sigma Way: How GE, Motorola And Other Top
Companies Are Honing Their Performance (McGrawHill, New York, 2000).

32. R. Banuelas and J. Antony, Going From Six-Sigma to Design for Six-Sigma: An Exploratory Study Using Analytic Hierarchy Process, The TQM Mag. 15(5) (2003) 334-344.

33. R. Banuelas, C. Tennant, I. Tuersley and S. Tang, Selection of Six-Sigma Projects in the UK, The TQM Mag. 18(5) (2006) 514-527.

34. R.B. Coronado and J. Antony, Critical Success Factors for the Successful Implementation of Six Sigma Projects in Organizations, The TQM Mag. 14(2) (2002)92-99.

35. S. Önüt, and S. Soner, Transshipment site selection using the AHP and TOPSIS approaches under fuzzy environment, Waste Manage. 28(9) (2008)1552-1559.

36. S. Önüt, S.S. Kara and T. Efendigil, A Hybrid Fuzzy MCDM Approach To Machine Tool Selection, $J$ Intell Manuf. 19(4) (2008) 443-453.

37. S. Önüt, S.S. Kara, and E. Işik, Long Term Supplier Selection Using a Combined Fuzzy MCDM Approach: A Case Study for a Telecommunication Company, Expert Syst Appl. 36(2) (2009) 3887-3895.

38. S.H. Tsaur, T.Y. Chang and C.H. Yen, The Evaluation of Airline Service Quality By Fuzzy MCDM, Tourism Manage. 23(2) (2002) 107-115.

39. S.J. Chen and C.L. Hwang, Fuzzy Multiple Attribute Decision Making Methods and Applications, (SpringerVerlag, Berlin, 1992).

40. T. Pyzdek, Six Sigma and Beyond: Selecting Six Sigma Projects, Quality Digest, Available at: http://www.qualitydigest.com/sept00/html/sixsigma.html. (Accessed November 2008)

41. T. Pyzdek, The Six Sigma Project Planner, (McGrawHill, New York, 2003)

42. T. Yang, and C.C. Hung, Multiple-Attribute Decision Making Methods for Plant Layout Design Problem, Robot Cim-Int Manuf. 23(1) (2007) 126-137.

43. T.L. Saaty, How to Make a Decision: The Analytic Hierarchy Process, Interfaces, 24(6) (1994) 19-43.

44. T.L. Saaty, The Analytic Hierarchy Process, 3rd edn. (McGraw- Hill, New York, 1980).

45. U.D. Kumar, H. Saranga, J.E. Ramirez-Marquez and D. Nowicki, Six Sigma Project Selection Using Data Envelopment Analysis, The TQM Mag. 19(5) (2007) 419441.

46. Y.H. Kwak and F.T. Anbari, Benefits, Obstacles and Future of Six Sigma Approach, Technovation, 26(5/6) (2006) 708-715. 